\title{
Towards simulating a realistic data analysis with an optimised angular power spectrum of spectroscopic galaxy surveys
}

\author{
Guglielmo Faggioli ${ }^{1}$, Konstantinos Tanidis ${ }^{1,2}$ (D) and Stefano Camera ${ }^{1,2,3}$ \\ ${ }^{1}$ Dipartimento di Fisica, Università degli Studi di Torino, via P. Giuria 1, 10125 Torino, Italy, ${ }^{2}$ INFN, Sezione di Torino, via \\ P. Giuria 1, 10125 Torino, Italy, and ${ }^{3}$ INAF, Osservatorio Astrofisico di Torino, strada Osservatorio 20, 10025 Pino \\ Torinese, Italy \\ Corresponding author. E-mail: tanidis@to.infn.it
}

(Received 27 July 2020; Revised 23 October 2020; Accepted 26 October 2020)

\begin{abstract}
The angular power spectrum is a natural tool to analyse the observed galaxy number count fluctuations. In a standard analysis, the angular galaxy distribution is sliced into concentric redshift bins and all correlations of its harmonic coefficients between bin pairs are considered-a procedure referred to as 'tomography'. However, the unparalleled quality of data from oncoming spectroscopic galaxy surveys for cosmology will render this method computationally unfeasible, given the increasing number of bins. Here, we put to test against synthetic data a novel method proposed in a previous study to save computational time. According to this method, the whole galaxy redshift distribution is subdivided into thick bins, neglecting the cross-bin correlations among them; each of the thick bin is, however, further subdivided into thinner bins, considering in this case all the cross-bin correlations. We create a simulated data set that we then analyse in a Bayesian framework. We confirm that the newly proposed method saves computational time and gives results that surpass those of the standard approach.
\end{abstract}

Key words: cosmology; theory; large-scale structure of the Universe; observations; cosmological parameters

\section{Introduction}

The forthcoming generation of experiments targeting the large-scale cosmic structure will provide us with data of exquisite quality, from which it will be possible to extract cosmological information to test the our current cosmological model $(\Lambda \mathrm{CDM})$, for instance investigating the nature of dark energy and dark matter. The two main probes envisaged for such experiments are weak gravitational lensing and galaxy clustering. In this paper, we shall focus on the latter.

Forthcoming galaxy surveys, such as the Euclid satellite (Amendola et al., 2013, 2018; Laureijs et al., 2011), the Legacy Survey of Space and Time (LSST Science Collaboration et al., 2009), and the Square Kilometre Array (Bacon et al., 2020), will be characterised by a high computational time cost in their analysis, motivating the search for new optimised methods. For this reason, this work aims at developing an improved analysis technique, taking inspiration from Camera et al. (2018). In particular, we adopt the philosophy there presented and implement it in a likelihood-based approach, simulating a synthetic data set that we then fit against the theoretical model predictions.

\footnotetext{
(C) The Author(s), 2020. Published by Cambridge University Press. This is an Open Access article, distributed under the terms of the Creative Commons Attribution licence (http://creativecommons.org/licenses/by/4.0/), which permits unrestricted re-use, distribution, and reproduction in any medium, provided the original work is properly cited.
} 
This paper is outlined as follows. In Section 2, we introduce the survey assumptions considered throughout our analysis, present the harmonic-space angular power spectrum for galaxy clustering, describe in detail the optimised method, and show the likelihood and the scale cuts applied for the analysis. In Section 3, we discuss the results obtained with the standard and the optimised method. Finally, conclusions are presented in Section 4.

\section{Methods}

\subsection{Survey assumptions}

We adopt the same survey specifications of Camera et al. (2018, see their Section 2.2 and references therein, for details), who first proposed the method and tested it via a Fisher matrix analysis. Specifically, we consider a spectroscopic galaxy survey targeting $\mathrm{H} \alpha$ emitters in the redshift range between 0.6 and 2 , with an accuracy that can be modelled with a redshift-dependent Gaussian uncertainty on the distribution on the measured redshift with width $\sigma_{z}=0.001(1+z)$. The linear galaxy bias is modelled as $b(z)=\sqrt{(1+z)}$.

\subsection{The harmonic-space galaxy power spectrum}

The harmonic-space (also, angular) power spectrum represents the natural tool to probe fluctuations in the observed galaxy distribution as measured from our point of view as observers. For large multipole values, $\ell \gg 1$, it is possible to employ the Limber approximation (Kaiser, 1992; Limber, 1953), to reduce the computational effort thanks to its collapsing a three-dimensional integral into a one-dimensional one. Under this assumption, the theoretical power spectrum of galaxy number counts for the redshift bin pair $i-j$ and on linear scales reads

$$
C_{\ell}^{i j}=\int \frac{\mathrm{d} \chi}{\chi^{2}} W^{i}(\chi) W^{j}(\chi) P_{\operatorname{lin}}\left(k=\frac{\ell+1 / 2}{\chi}\right),
$$

where $\chi(z)$ is the comoving distance to redshift $z$,

$$
W^{i}(\chi)=n^{i}(\chi) b(\chi) D(\chi)
$$

is the window function in the $i$ th redshift bin, with $n^{i}(\chi)$ its normalised galaxy distribution, $b(\chi)$ is the linear galaxy bias, and $D(\chi)$ the linear growth factor. Finally, $P_{\text {lin }}(k)$ is the linear matter power spectrum at $z=0$, which is here provided by the Boltzmann solver CAMB (Lewis et al., 2000).

It is worth noting that the observed clustering of galaxies contains other terms on top of what we have described above, which is due to perturbations in the underlying matter density distribution (Bonvin \& Durrer, 2011; Challinor \& Lewis, 2011). The most notable of such terms are redshift-space distortions (RSD) and lensing magnification. ${ }^{1}$ However, these terms are suppressed on the scales of interest in this analysis and for the bin sizes we adopt, meaning we can safely neglect to include them.

\subsection{The traditional approach}

On the one hand, data from spectroscopic galaxy surveys has customarily been analysed in terms of the Fourier-space power spectrum and its decomposition into Legendre multipoles. Whilst this approach has worked perfectly, for the redshift and sky coverages of data hitherto collected, it is arguable that some of its underlying assumptions will no longer be met with the next generation of cosmological experiments (see e.g. Blake et al., 2018; Ruggeri et al., 2018). Moreover, the fluctuations in the observed galaxy number counts

\footnotetext{
${ }^{1}$ For a window function accounting for other terms on top of the density field in the Limber approximation we refer the reader to the relevant literature (see Tanidis et al., 2019; Chisari et al., 2019).
} 
contain terms that cannot be decomposed in Fourier modes, like the nonlocal contribution from gravitational lensing, which will be all the more important for deeper surveys (Camera et al., 2015; Cardona et al., 2016).

On the other hand, the standard tomographic approach for the computation of the galaxy angular power spectrum $C_{\ell}^{i j}$ is based on all correlations among bin pairs $i-j$ across the whole redshift range. Now, the benchmark survey described in Section 2.1 will easily be able to slice the observed galaxy distribution in bins of width $\sim 0.01$, which, for the redshift range considered, results into about $10^{4}$ between auto- and cross-bin correlations. Such a number has to be further multiplied by the number of bins in multipole space the data will be binned into. This is clearly computationally unfeasible, in the prospect of a likelihood-based analysis scanning — at the very least—-the six-dimensional parameter space of the 'vanilla' $\Lambda$ CDM model.

\subsection{The new method}

This conundrum motivates the research of new strategies to analyse forthcoming surveys data sets. Among the different proposals, we follow Camera et al. (2018), who proposed to combine relevant aspects of the two standard techniques described above. Fourier-space analyses usually employ a thick redshift binning, e.g. with width $\Delta z \approx 0.1$; all Fourier modes inside the bin are then considered, but the correlations among adjacent $z$-bins are not taken into account. However, applying this approach facevalue to the harmonic-space $C_{\ell}^{i j}$ means losing information by squashing all the galaxies within the relatively large $\Delta z$ bin onto a single redshift slice.

Hence, the idea is to combine the two approaches in a 'hybrid' method. This method is characterised by two binning tiers: the galaxy distribution is binned by adopting a set of top-hat thick bins; each of these is further binned into top-hat thin bins, convolved with a Gaussian in order to take into account for the small although non-negligible errors in the spectroscopic redshift estimation. This division is made by using equi-spaced bins. Each thick bin is considered as an independent survey, hence cross-correlation between them is not computed, while it is for the thin bins inside the thick ones. The resulting tomographic matrix $C_{\ell}^{i j}$ is thus block diagonal by construction.

In this paper, we include two hybrid binning configurations in the same redshift range $z \in[0.6,2.0]$, both smoothed by a Gaussian with $\sigma_{z}=0.001$ :

1. 7 equi-spaced thick bins of redshift width $\Delta z=0.2$, each having 5 equi-spaced thin bins of width $\delta z$ $=0.04$. This case is represented by black and coloured bins, respectively, in the left panel of Figure 1 ;

2. 10 equi-spaced thick bins of redshift width $\Delta z=0.14$, each having 7 equi-spaced thin bins of width $\delta z=0.02$, as shown in the right panel of Figure 1.
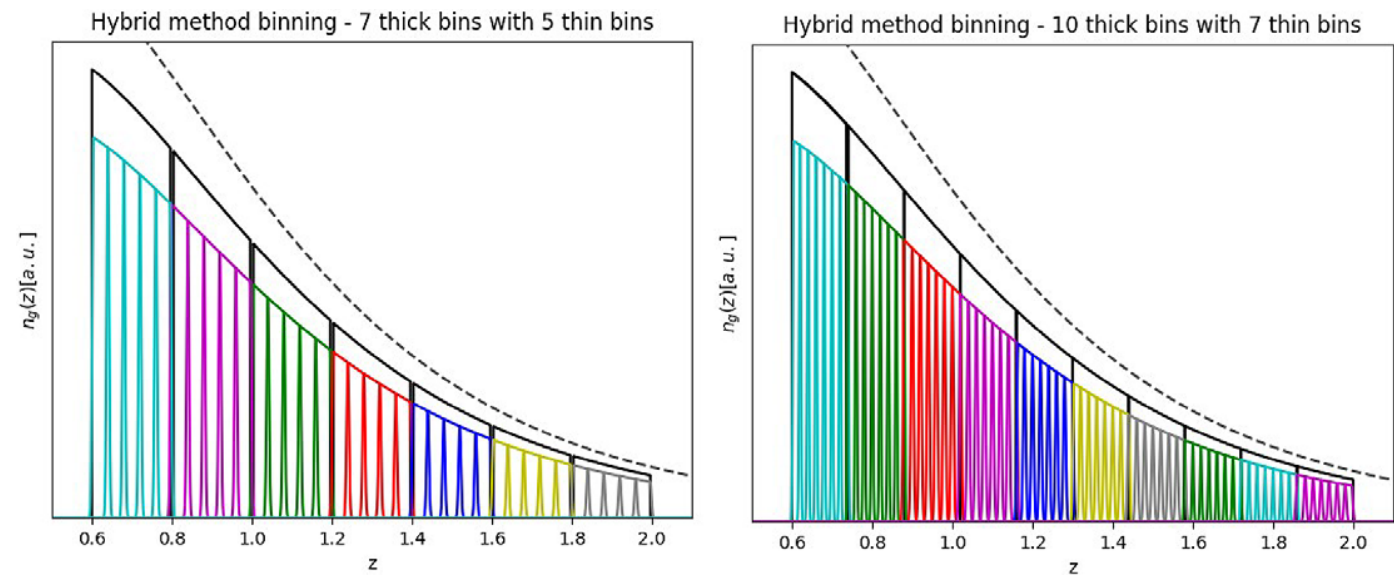

Figure 1. The top dashed black curve shows the unbinned galaxy distribution, $n_{\mathrm{g}}(z)$. Black curves correspond to thick bins, whilst coloured ones to thin bins inside each thick bin. (To enhance readability we have rescaled all the distributions by arbitrary factors.) 


\subsection{Set-up of statistical analysis}

To construct the likelihood and forecast constraints on the cosmological parameters of interest, we employ the publicly available suite CosmoSIS (Zuntz et al., 2015), which we modify to reproduce the hybrid method described above. For our synthetic data, we choose as a reference a flat $\Lambda \mathrm{CDM}$ model with the cosmological parameter set $\boldsymbol{\theta}=\left\{\Omega_{\mathrm{m}}, h, \Omega_{\mathrm{b}}, n_{\mathrm{s}}, \ln \left(10^{10} A_{\mathrm{s}}\right)\right\}$, with fiducial values $\boldsymbol{\theta}_{\text {fid }}=\{0.31,0.6774$, $0.05,0.9667,3.06\}$ using the angular power spectra as given in Equation 1. For details on the samplers and analysis employed to explore the parameter space, see Section 3.

For the data, we assume a Gaussian likelihood, and we focus on minimising the chisquared. In other words, we do not include the likelihood normalisation in the parameter estimation. This assumption does not hinder our result, as the data covariance is assumed independent of the cosmological parameters.

Concerning the covariance of the galaxy clustering signal given in Equation 1, we adopt the Gaussian approximation, namely

$$
\operatorname{Cov}\left(C_{\ell}^{i j}, C_{\ell^{\prime}}^{m n}\right)=\frac{\delta_{\mathrm{K}}^{\ell \ell^{\prime}}}{2 \ell \Delta \ell f_{\text {sky }}}\left[\left(C_{\ell}^{i m}+\frac{\delta_{\mathrm{K}}^{i m}}{\bar{n}_{i}}\right)\left(C_{\ell}^{j n}+\frac{\delta_{\mathrm{K}}^{j n}}{\bar{n}_{j}}\right)+\left(C_{\ell}^{i n}+\frac{\delta_{\mathrm{K}}^{i n}}{\bar{n}_{i}}\right)\left(C_{\ell}^{j m}+\frac{\delta_{\mathrm{K}}^{j m}}{\bar{n}_{j}}\right)\right],
$$

where $\Delta \ell$ is the multipole binning width, $f_{\text {sky }}$ the sky fraction covered by the survey, $\delta_{\mathrm{K}}$ the Kronecker symbol and $\bar{n}_{i}$ is the surface galaxy density in bin $i$.

The angular power spectra are computed with the Limber approximation and in the linear regime, we therefore focus on multipole range, $\ell \in[100,800]$ as a reasonable interval. It is possible that for a few binpair configurations either the lower or upper multipole limit exceeds the range of validity of the Limber approximation or the nonlinear scale. However, we do not aim to make forecasts for a specific experiment but rather to compare the performance of the standard and hybrid methods in a realistic setting, and thus this choice does not affect our conclusions. In both binning scenario we consider $n_{\ell}=5$ log-spaced multipole values in the aforementioned range.

\section{Results}

Here, we present and compare the results obtained with the standard and the hybrid methods. As already mentioned in Subsection 2.4, we applied two hybrid binning configurations in the redshift range $z \in[0.6,2.0]$. We can summarise our findings as follows. All cosmological parameter reconstructed mean values and inferred $68 \%$ confidence level intervals are summarised in Table 1.

1. For the standard approach we use 20 equi-populated bins in the redshift range $z \in[0.6,2.0]$, and $n=$ 5 multipole values in the considered $l$ range. We use the multinest sampler (Feroz et al., 2009) to forecast constraints.

Table 1. Summary of analysis results for each parameter (first column) with: its input fiducial value, $\theta_{\text {fid }}$ (second column); reconstructed mean value, $\theta$ (third, fifth, and seventh column); and $68 \%$ confidence level error interval, $\sigma_{\theta}$ (fourth, sixth, and eighth column).

\begin{tabular}{|c|c|c|c|c|c|c|c|}
\hline \multirow[b]{2}{*}{ Parameter } & \multirow[b]{2}{*}{$\theta_{\text {fid }}$} & \multicolumn{2}{|c|}{ Standard } & \multicolumn{2}{|c|}{ Hybrid (config. 1) } & \multicolumn{2}{|c|}{ Hybrid (config. 2) } \\
\hline & & $\theta$ & $\sigma_{\theta}$ & $\theta$ & $\sigma_{\theta}$ & $\theta$ & $\sigma_{\theta}$ \\
\hline$\Omega_{\mathrm{m}}$ & 0.31 & 0.310001 & 0.00015 & 0.310002 & 0.000074 & 0.310001 & 0.000023 \\
\hline$h$ & 0.6774 & 0.685 & 0.028 & 0.684 & 0.026 & 0.681 & 0.019 \\
\hline$\Omega_{\mathrm{b}}$ & 0.05 & 0.0511 & 0.0028 & 0.0505 & 0.0026 & 0.0499 & 0.0023 \\
\hline$n_{\mathrm{s}}$ & 0.9667 & 0.964 & 0.014 & 0.0964 & 0.013 & 0.965 & 0.011 \\
\hline $\ln \left(10^{10} A_{s}\right)$ & 3.06 & 3.048 & 0.017 & 3.049 & 0.015 & 3.053 & 0.012 \\
\hline
\end{tabular}


2. Regarding the first configuration of the hybrid binning we use equi-spaced thick bins with width $\Delta z=0.2$, in the same redshift range, while for the thin bins we use a width $\delta z=0.04$. This means that we have seven thick bins, considered as seven independent surveys, each of them containing five thin bins. Again, we use $n=5$ multipole values while for the sampling method we chose the emcee sampler (Foreman-Mackey et al., 2013), better suited for the tomographic matrix configuration of the hybrid method.

3. In the second hybrid binning configuration the thick bin width is $\Delta z=0.14$ and the thin bins $\delta z=$ 0.02 , working now with finer binning of 10 thick bins each containing 7 thin bins. The sampler employed is, again, emcee.

For a more thorough comparison of the two methods, in Table 2 we also show the computation times running a fixed cosmology on a specific parameter value set for the standard and the hybrid method. For sake of comparison of running time test, we consider a third hybrid binning with 14 thick bins each containing 10 thin bins while keeping the same smearing with the previous cases. It can be clearly seen from Table 2 that the larger the number of the bins, the more time we save by using the hybrid method with respect to the standard one.

Another major advantage of the hybrid method over the standard approach is that it yields tighter constraints on the parameter of interest. This is due to the fact that the finer binning of the thin bins allows us to recover partly the three-dimensional information encoded in the correlation of galaxies within the thick bin. To appreciate better the aforementioned enhancement in constraining power, in Figure 2 we show the ratio between the $68 \%$ marginal error intervals on each parameter from the hybrid method and the same obtained with the standard approach, for the two binning configurations of Subsection 2.4 (green and red candlesticks, for configuration 1 and 2 respectively). Note that the blue candlesticks are the ratio of the $68 \%$ marginal error intervals of the standard approach with themselves, simply to guide the reader's eye. This clearly shows us how the finer the binning, the tighter the

Table 2. Comparison between standard and hybrid computation times for a fixed cosmology.

\begin{tabular}{ccc} 
No. of bins & Standard method running time $[\mathrm{s}]$ & Hybrid method running time [s] \\
\hline 35 & 7 & 5 \\
\hline 70 & 14 & 8 \\
\hline 140 & 22 & 11 \\
\hline
\end{tabular}

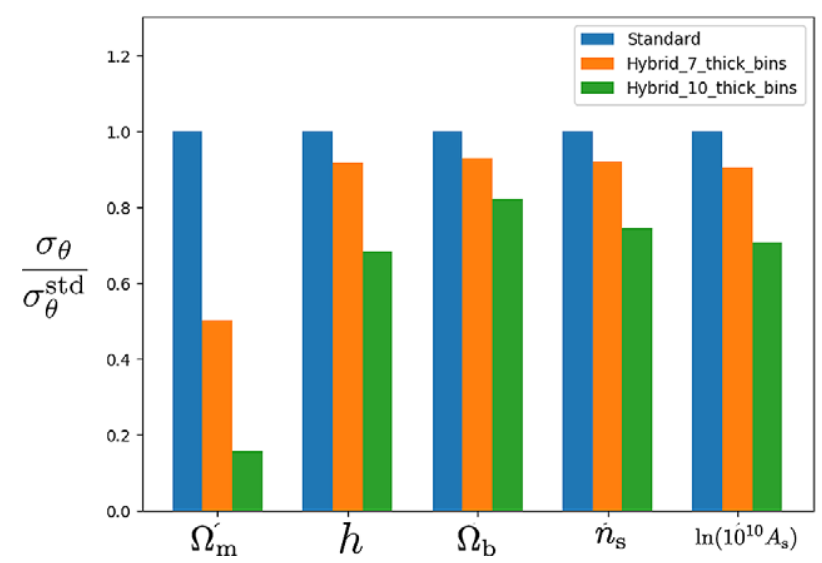

Figure 2. Comparison between marginalised errors from the hybrid methd, $\sigma_{\theta}$, and what obtained from the standard approach, $\sigma_{\theta}^{\text {std }}$, on the estimated cosmological parameters. 

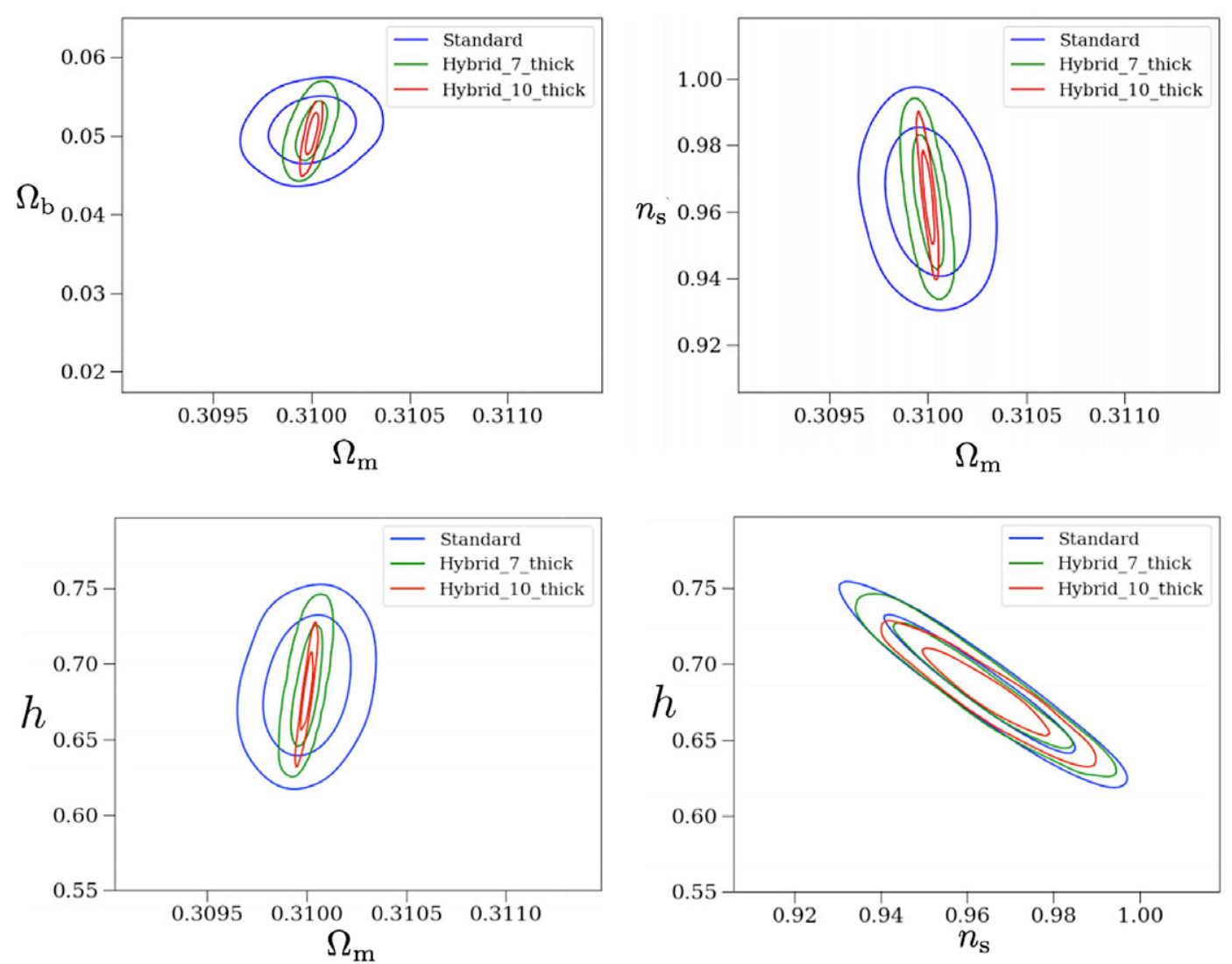

Figure 3. Two-dimensional joint posteriors on the following planes: $\Omega_{\mathrm{m}}-\Omega_{\mathrm{b}}$ (top left panel), $\Omega_{\mathrm{m}}-n_{\mathrm{s}}$ (top right panel), $\Omega_{\mathrm{m}}-h$ (bottom left panel) and $n_{\mathrm{s}}-h$ (bottom right panel). Inner contours represent the $68 \%$ confidence level areas, while the outer the $95 \%$ areas.

constraints, both because we can track better the cosmic growth and the redshift evolution of the source distribution (thick binning), and because we can recover radial information (thin binning). Actually, the fact that even the seven thick bins of the hybrid binning configuration no. 1 perform better than the 20 bins of the standard method is a proof that radial information within the bin is crucial for accurate cosmological parameter estimation.

To have a deeper understanding of the impact of the radial information retrieved by the hybrid approach, it is useful to look not only at the constraints on a single parameter, but rather at the cross-talks among different parameters, which tell us about intrinsic parameter degeneracies. Figure 3 shows the $68 \%$ and $95 \%$ joint marginal error contours on the two-dimensional parameter planes of the parameter set $\boldsymbol{\theta}$ for the three cases under investigation, i.e. the standard approach (blue contours), and the two binning configurations of the hybrid method (green and red contours for configuration 1 and 2 respectively). Looking at these plots it is evident that the new method is capable of constraining cosmological parameters better than the standard one, giving relative errors which are of the same order of magnitude but smaller. It is worth noting that the parameter $\Omega_{\mathrm{m}}$ is particularly better constrained with the hybrid procedure, having a relative error half of the one given by the standard method.

\section{Conclusions}

In this work we make forecasts to compare the constraining power and reliability of a new hybrid tomographic method with the standard tomographic approach generally applied in the studies of 
spectroscopic galaxy clustering. We perform this comparison in a likelihood-based Bayesian approach going beyond the Fisher matrix analysis. We confirm that the standard and hybrid methods give comparable results, but the latter appears to be more constraining. On top of that, it saves computational time, as shown in Table 2. However, several approximations are made: we do not consider the RSD or correction due to lensing magnification (which nonetheless should be subdominant for fine redshift slicing). Also, we do not account for nuisance parameters, which are considered in the original paper. Finally, we work in the Limber approximation and calculate the angular power spectra at an exiguous number of multipole values to speed up the analysis computation. This, in principle, is not an issue, but in future works the hybrid method should be tested with finer binning, both in angular and in redshift space. Consequently, these approximations do not allow for a face-value comparison with the original paper results. In a forthcoming work we plan to reproduce the same analysis using finer binning, and introducing nuisance parameters as well as the contributions from RSD and magnification on the galaxy density field.

Author contributions. SC conceived the methodology. KT and GF created the algorithm. GF performed the analysis. SC and KT supervised the analysis. GF, KT, and SC wrote the article.

Funding information. The authors wish to thanks Roy Maartens and José Fonseca for useful feedback on an early draft of this paper, as well as the referees who helped us improving the presentation of our results. The authors acknowledge support from the 'Departments of Excellence 2018-2022' Grant (L. 232/2016) awarded by the Italian Ministry of Education, University and Research (miur). SC also acknowledges support by miur through Rita Levi Montalcini project 'prometheus - Probing and Relating Observables with Multi-wavelength Experiments To Help Enlightening the Universe's Structure' in the early stages of this project.

Conflicts of interest. GF, KT, and SC declare none.

Data availability. Data sharing not applicable - no new data generated.

\section{References}

Amendola, L., Appleby, S., Bacon, D., Baker, T., Baldi, M., Bartolo, N., Blanchard, A., Bonvin, C., Borgani, S., Branchini, E., Burrage, C., Camera, S., Carbone, C., Casarini, L., Cropper, M., de Rham, C., Di Porto, C., Ealet, A., Ferreira, P. G., Zlosnik, T., ..., The Euclid Theory Working Group. (2013). Cosmology and fundamental physics with the Euclid satellite. Living Reviews in Relativity, 16, 6.

Amendola, L., Appleby, S., Bacon, D., Baker, T., Baldi, M., Bartolo, N., Blanchard, A., Bonvin, C., Borgani, S., Branchini, E., Burrage, C., Camera, S., Carbone, C., Casarini, L., Cropper, M., de Rham, C., Di Porto, C., Ealet, A., Ferreira, P. G., Zlosnik, T., ..., The Euclid Theory Working Group. (2018). Cosmology and fundamental physics with the Euclid satellite. Living Reviews in Relativity, 21, 2.

Bacon, D. J., Battye, R. A., Bull, P., Camera, S., Ferreira, P. G., Harrison, I., Parkinson D., Pourtsidou A, Santos M. G., Wolz, L., Abdalla, F., Akrami, Y., Alonso, D., Andrianomena, S., Ballardini, M., Bernal, J. L., Bertacca, D., Bengaly, C. A. P., Bonaldi, A., ... Zuntz, J. (2020). Cosmology with phase 1 of the Square Kilometre Array Red Book 2018: Technical specifications and performance forecasts. Publications of the Astronomical Society of Australia, 37, e007.

Blake, C., Carter, P., \& Koda, J. (2018). Power spectrum multipoles on the curved sky: An application to the 6-degree Field Galaxy Survey. Monthly Notices of the Royal Astronomical Society, 479, 5168-5183.

Bonvin, C., \& Durrer, R. (2011). What galaxy surveys really measure. Physical Review D, 84, 063505.

Camera, S., Fonseca, J., Maartens, R., \& Santos, M. G. (2018). Optimized angular power spectra for spectroscopic galaxy surveys. Monthly Notices of the Royal Astronomical Society, 481, 1251.

Camera, S., Raccanelli, A., Bull, P., Bertacca, D., Chen, X., Ferreira, P. G., Kunz, M., Maartens, R., Mao, Y., Santos, M. G., Shapiro, P. R., Viel, M., \& Xu, Y. (2015). Cosmology on the largest scales with the SKA. Proceedings of Advancing Astrophysics with the Square Kilometre Array PoS(AASKA14), 25.

Cardona, W., Durrer, R., Kunz, M., \& Montanari, F. (2016). Lensing convergence and the neutrino mass scale in galaxy redshift surveys. Physical Review D, 94, 043007.

Challinor, A., \& Lewis, A. (2011). Linear power spectrum of observed source number counts. Physical Review D, 84, 043516.

Chisari, N. E., Alonso, D., Krause, E., Leonard, C. D., Bull, P., Neveu, J., Villarrea, A., Singh, S, McClintock, T., Ellison, J., Du, Z., Zuntz, J., Mead, A., Joudaki, S., Lorenz, C. S., Tröster, T., Sanchez, J., Lanusse, F., Ishak, M., ... Wagoner, E. L. (2019). Core cosmology library: Precision cosmological predictions for LSST. The Astrophysical Journal Supplement Series, $242,2$. 
Feroz, F., Hobson, M. P., \& Bridges, M. (2009). MultiNest: An efficient and robust Bayesian inference tool for cosmology and particle physics. Monthly Notices of the Royal Astronomical Society, 398, 1601.

Foreman-Mackey, D., Hogg, D. W., Lang, D., \& Goodman, J. (2013). emcee: The MCMC hammer. Publications of the Astronomical Society of the Pacific, 125, 306.

Kaiser, N. (1992). Weak gravitational lensing of distant galaxies. The Astrophysical Journal, 388, 272.

Laureijs, R., Amiaux, J., Arduini, S., Auguères, J.-L., Brinchmann, J., Cole, R., Cropper, M., Dabin, C., Duvet, L., Ealet, A., Garilli, B., Gondoin, P., Guzzo, L., Hoar, J., Hoekstra, H., Holmes, R., Kitching, T., Maciaszek, T., Mellier, Y., ... Zucca, E. (2011). Euclid definition study report.

Lewis, A., Challinor, A., \& Lasenby, A. (2000). Efficient computation of cosmic microwave background anisotropies in closed Friedmann-Robertson-Walker models. The Astrophysical Journal, 538, 473

Limber, D. N. (1953). The analysis of counts of the extragalactic nebulae in terms of a fluctuating density field. The Astrophysical Journal, 117, 134.

LSST Science Collaboration, Abell, P. A., Allison, J., Anderson, S. F., Andrew, J. R., Angel, J. R. P., Armus, L., Arnett, D., Asztalos, S. J., Axelrod, T. S., Bailey, S., Ballantyne, D. R., Bankert, J. R., Barkhouse, W. A., Barr, J. D., Barrientos, L. F., Barth, A. J., Bartlett, J. G., Becker, A. C., Becla, J., ... Zhan, H. (2009). Lsst science book, version 2.0.

Ruggeri, R., Percival, W. J., Gil-Marín, H., Beutler, F., Mueller, E.-M., Zhu, F., Padmanabhan, N., Zhao, G.-B., Zarrouk, P., Sánchez, A. G., Bautista, J., Brinkmann, J., Brownstein, J. R., Baumgarten, F., Chuang, C.-H., Dawson, K., Seo, H.-J., Tojeiro, R., \& Zhao, C. (2018). The clustering of the SDSS-IV extended Baryon Oscillation Spectroscopic Survey DR14 quasar sample: Measuring the evolution of the growth rate using redshift-space distortions between redshift 0.8 and 2.2 . Monthly Notices of the Royal Astronomical Society, 483, 3878-3887.

Tanidis, K., Camera, S., \& Parkinson, D. (2019). Developing a unified pipeline for large-scale structure data analysis with angular power spectra-II. A case study for magnification bias and radio continuum surveys. Monthly Notices of the Royal Astronomical Society, 491, 4869.

Zuntz, J., Paterno, M., Jennings, E., Rudd, D., Manzotti, A., Dodelson, S., Bridle, S., Sehrish, S., \& Kowalkowski, J. (2015). CosmoSIS: Modular cosmological parameter estimation. Astronomy and Computing,12, 45.

Cite this article: Faggioli G, Tanidis K, Camera S (2020). Towards simulating a realistic data analysis with an optimised angular power spectrum of spectroscopic galaxy surveys Experimental Results, 1, e54, 1-11. https://doi.org/10.1017/exp.2020.59 


\title{
Peer Reviews
}

\author{
Reviewing editor: Dr. Jackson Levi Said \\ University of Malta, Msida, Malta
}

This article has been accepted because it is deemed to be scientifically sound, has the correct controls, has appropriate methodology and is statistically valid, and has been sent for additional statistical evaluation and met required revisions.

doi:10.1017/exp.2020.59.pr1

\section{Review 1: Optimised angular power spectrum for spectroscopic galaxy surveys: a Bayesian approach}

\author{
Reviewer: Dr. Benjamin Bose \\ University of Geneva, Physics, Quai Ernest-Ansermet 24, Geneve, Switzerland, 1205
}

Date of review: 05 August 2020

\begin{abstract}
(C) The Author(s), 2020. Published by Cambridge University Press This is an Open Access article, distributed under the terms of the Creative Commons Attribution licence (http://creativecommons.org/licenses/by/4.0/), which permits unrestricted re-use, distribution, and reproduction in any medium, provided the original work is properly cited.
\end{abstract}

Conflict of interest statement. Reviewer declares none

Comments to the Author: There are two avenues that the authors presumably wish to take, the first being a clear comparison with SC18 when using the more robust Bayesian analyses. This is made difficult from the get-go because RSD is omitted. The second possible avenue is to provide a second test of the standard and hybrid approaches in the context of a Bayesian analysis. In this case, how do the authors justify not using a non-linear power spectrum in equation.1 such as halofit? If nuisance (e.g. bias) parameters are not considered as hinted at in Section.4, then it seems there will be no consistency issues by using the pure dark matter halofit formula in equation 1.

In summary, it is not clear what additional information they are they providing over the Fisher analysis of SC18 by using linear theory for model and data as well as a Gaussian covariance. I feel the authors should either revise the analysis or make it very clear what the goal is, and argue clearly against extensions, such as (given their methodology) to using non-linear spectra. I also have some minor comments:

Further, the authors should describe explicitly how the synthetic data is created (presumably using linear theory) and they should comment explicitly that bias is neglected (if that is the case).

The label font of figure 1 could do with increasing.

Could figures 3 and 4 be combined?

Bacon et al 2018 needs updating and Mon.Not.Roy.Astron.Soc to be used consistently.

\section{Score Card}

Presentation

Does the paper cite relevant and related articles appropriately? (30\%) 
Does the abstract correctly embody the content of the article? (25\%)

Does the introduction give appropriate context? (25\%)

Is the objective of the experiment clearly defined? (25\%)

Analysis

Does the discussion adequately interpret the results presented? (40\%)

Is the conclusion consistent with the results and discussion? (40\%)

Are the limitations of the experiment as well as the contributions of the experiment clearly outlined? (20\%) 


\section{Review 2: Optimised angular power spectrum for spectroscopic galaxy surveys: a Bayesian approach}

Reviewer: Prof. Rafael Nunes

Date of review: 17 October 2020

(C) The Author(s), 2020. Published by Cambridge University Press This is an Open Access article, distributed under the terms of the Creative Commons Attribution licence (http://creativecommons.org/licenses/by/4.0/), which permits unrestricted re-use, distribution, and reproduction in any medium, provided the original work is properly cited.

Conflict of interest statement. I dont have any competing personal, professional or financial interests in my evaluation of the work under review.

Comments to the Author: The authors introduce a computational alternative to calculate the power spectrum of galaxy clustering. Table I and Figure 2 summary the proposed methodology. In special, the hybrid method presented in the article seems to provide tighter constraints on the baseline parameters when compared to the standard approach. This point is very interesting and are the main aspects of the work. After fix the minor points below, the paper can be accepted for publication in Experimental Results. 1 - It would be interesting to consider a minimal extension of the LCDM, such as w + LCDM or M_\{mu + N_eff + LCDM, in light of perspectives as in Fig. 2. These case are minimal parameters the great interest in light of the perspective of future surveys.

2 - The authors applied the methodology in the range $\mathrm{z}$ in $[0.6,2.0]$ and $\mathrm{l}$ in $[100,800]$. By not considering non-linear effects in modeling, has this effects on the estimates summarized in Fig. 2 ? in particular on IOmega_m.

\section{Score Card}

Presentation

Does the paper cite relevant and related articles appropriately? (30\%)

\section{Context}

Does the abstract correctly embody the content of the article? (25\%)

Does the introduction give appropriate context? (25\%)

Is the objective of the experiment clearly defined? (25\%)

Analysis

Are the limitations of the experiment as well as the contributions of the experiment clearly outlined? $(20 \%)$ 